\title{
Improved cartilage regeneration by implantation of acellular biomaterials after bone marrow stimulation: a systematic review and meta-analysis of animal studies
}

\author{
Michiel W Pot ${ }^{1}$, Veronica K Gonzales $^{2}$ ， Pieter Buma ${ }^{2}$, Joanna IntHout ${ }^{3}$ ， Toin H van Kuppevelt ${ }^{1}$, Rob BM de \\ Vries ${ }^{4}$, Willeke F Daamen ${ }^{\text {Corresp. } 1}$ \\ 1 Department of Biochemistry, Radboud Institute for Molecular Life Sciences, Radboud university medical center, Nijmegen, The Netherlands \\ 2 Department of Orthopedics, Radboud Institute for Molecular Life Sciences, Radboud university medical center, Nijmegen, The Netherlands \\ 3 Radboud Institute for Health Sciences, Department for Health Evidence, Radboud university medical center, Nijmegen, The Netherlands \\ 4 SYRCLE (SYstematic Review Centre for Laboratory animal Experimentation), Central Animal Laboratory, Radboud university medical center, Nijmegen, \\ The Netherlands \\ Corresponding Author: Willeke F Daamen \\ Email address: Willeke.Daamen@radboudumc.nl
}

Microfracture surgery may be applied to treat cartilage defects. During the procedure the subchondral bone is penetrated, allowing bone marrow-derived mesenchymal stem cells to migrate towards the defect site and form new cartilage tissue. Microfracture surgery generally results in the formation of mechanically inferior fibrocartilage. As a result, this technique offers only temporary clinical improvement. Tissue engineering and regenerative medicine may improve the outcome of microfracture surgery. Filling the subchondral defect with a biomaterial may provide a template for the formation of new hyaline cartilage tissue. In this study, a systematic review and meta-analysis were performed to assess the current evidence for the efficacy of cartilage regeneration in preclinical models using acellular biomaterials implanted after marrow stimulating techniques (microfracturing and subchondral drilling) compared to the natural healing response of defects. The review aims to provide new insights into the most effective biomaterials, to provide an overview of currently existing knowledge, and to identify potential lacunae in current studies to direct future research. A comprehensive search was systematically performed in PubMed and EMBASE (via OvidSP) using search terms related to tissue engineering, cartilage and animals. Primary studies in which acellular biomaterials were implanted in osteochondral defects in the knee or ankle joint in healthy animals were included and study characteristics tabulated (283 studies out of 6688 studies found). For studies comparing non-treated empty defects to defects containing implanted biomaterials and using semi-quantitative histology as outcome measure, the risk of bias (135 studies) was assessed and outcome data were collected for meta-analysis (151 studies). Random-effects meta-analyses were performed, using cartilage regeneration as 
outcome measure on an absolute $0-100 \%$ scale. Implantation of acellular biomaterials significantly improved cartilage regeneration by $15.6 \%$ compared to non-treated empty defect controls. The addition of biologics to biomaterials significantly improved cartilage regeneration by $7.6 \%$ compared to control biomaterials. No significant differences were found between biomaterials from natural or synthetic origin or between scaffolds, hydrogels and blends. No noticeable differences were found in outcome between animal models. The risk of bias assessment indicated poor reporting for the majority of studies, impeding an assessment of the actual risk of bias.

In conclusion, implantation of biomaterials in osteochondral defects improves cartilage regeneration compared to natural healing, which is further improved by the incorporation of biologics. 
1 Improved cartilage regeneration by implantation of acellular biomaterials after

2 bone marrow stimulation: a systematic review and meta-analysis of animal

3 studies

4 Michiel W. Pot ${ }^{1}$, Veronica K. Gonzales ${ }^{2}$, Pieter Buma ${ }^{2}$, Joanna IntHout ${ }^{3}$, Toin H. van Kuppevelt ${ }^{1 \#}$,

5 Rob B.M. de Vries ${ }^{4 \#}$, Willeke F. Daamen ${ }^{1 *}$

$6 \quad{ }^{1}$ Department of Biochemistry, Radboud Institute for Molecular Life Sciences, Radboud university

7 medical center, PO Box 9101, 6500 HB Nijmegen, The Netherlands

$8 \quad{ }^{2}$ Department of Orthopedics, Radboud Institute for Molecular Life Sciences, Radboud university

9 medical center, PO Box 9101, 6500 HB Nijmegen, The Netherlands

$10{ }^{3}$ Department for Health Evidence, Radboud university medical center, PO Box 9101, 6500 HB

11 Nijmegen, The Netherlands

$12{ }^{4}$ SYRCLE (SYstematic Review Centre for Laboratory animal Experimentation), Central Animal

13 Laboratory, Radboud university medical center, PO Box 9101, 6500 HB Nijmegen, The

14 Netherlands

15 " Contributed equally

16

$17{ }^{*}$ Corresponding author.

18 Willeke Daamen ${ }^{1}$

19 Radboud Institute for Molecular Life Sciences, Radboud university medical center, PO Box 9101,

206500 HB Nijmegen, The Netherlands

21 E: Willeke.Daamen@radboudumc.nl, T: +31 24361 0557, Fax: +31 243540339 


\section{Abstract}

Microfracture surgery may be applied to treat cartilage defects. During the procedure the subchondral bone is penetrated, allowing bone marrow-derived mesenchymal stem cells to migrate towards the defect site and form new cartilage tissue. Microfracture surgery generally results in the formation of mechanically inferior fibrocartilage. As a result, this technique offers only temporary clinical improvement. Tissue engineering and regenerative medicine may improve the outcome of microfracture surgery. Filling the subchondral defect with a biomaterial may provide a template for the formation of new hyaline cartilage tissue. In this study, a systematic review and meta-analysis were performed to assess the current evidence for the efficacy of cartilage regeneration in preclinical models using acellular biomaterials implanted after marrow stimulating techniques (microfracturing and subchondral drilling) compared to the natural healing response of defects. The review aims to provide new insights into the most effective biomaterials, to provide an overview of currently existing knowledge, and to identify potential lacunae in current studies to direct future research. A comprehensive search was systematically performed in PubMed and EMBASE (via OvidSP) using search terms related to tissue engineering, cartilage and animals. Primary studies in which acellular biomaterials were implanted in osteochondral defects in the knee or ankle joint in healthy animals were included and study characteristics tabulated (283 studies out of 6688 studies found). For studies comparing non-treated empty defects to defects containing implanted biomaterials and using semi-quantitative histology as outcome measure, the risk of bias (135 studies) was assessed and outcome data were collected for meta-analysis (151 studies). Random-effects meta-analyses were performed, using cartilage regeneration as outcome measure on an absolute 0-100\% scale. Implantation of acellular biomaterials significantly improved cartilage regeneration by $15.6 \%$ compared to non-treated empty defect controls. The addition of biologics to biomaterials significantly improved cartilage regeneration by $7.6 \%$ compared to control biomaterials. No significant differences were found between biomaterials from natural or synthetic origin or between scaffolds, hydrogels and blends. No noticeable differences were found in outcome between animal models. The risk of bias assessment indicated poor reporting for the majority of studies, impeding an assessment of the actual risk of bias. 
51 In conclusion, implantation of biomaterials in osteochondral defects improves cartilage 52 regeneration compared to natural healing, which is further improved by the incorporation of 53 biologics. 


\section{Introduction}

Articular cartilage is a specialized tissue that covers joint surfaces and provides a low-friction and load-bearing surface for a smooth motion of joints. The structure and function of the tissue can be compromised by traumatic injuries and degenerative joint diseases. Due to its avascular nature, damaged cartilage tissue does not heal spontaneously and it remains a challenge to fully restore tissue function (Ahn et al. 2009; Cao et al. 2012).

The surgical options to treat patients with a localized cartilage defect are limited to cartilage regeneration approaches such as autologous chondrocyte implantation and microfracture surgery (Aulin et al. 2013; Bal et al. 2010). The latter strategy, also known as bone marrow stimulation, is relatively simple, minimally invasive and inexpensive. During this procedure the subchondral bone plate below the cartilage lesion is perforated to initiate bleeding and induce a reparative response. The principle behind this regenerative resurfacing strategy is the migration of non-differentiated bone marrow-derived multipotent stem cells from the subchondral bone into the defect site leading to the formation of new cartilage tissue (Buma et al. 2003; de Mulder et al. 2014; Erggelet et al. 2009). Patients treated with bone marrow stimulation generally show clinical improvements up to 1.5 - 3 years after surgery. However, five years after surgery higher incidences of clinical failures are observed (Hoemann et al. 2010; van der Linden et al. 2013). The newly formed tissue generally consists of fibrocartilage repair tissue rather than hyaline cartilage, has limited filling of the defect, integrates poorly with the surrounding tissue and has inferior mechanical properties compared to hyaline cartilage (Dai et al. 2014). Therefore, the need for regeneration of more durable cartilage tissue persists.

Regenerative medicine and tissue engineering may offer promising alternatives and/or additions to clinical strategies that aim to restore damaged cartilage tissue. The construction of biomaterials and the incorporation of cells and biologics in these implants have been widely investigated for this purpose. Biomaterials can be implanted in osteochondral defects created by applying marrow stimulating techniques (microfracture and subchondral drilling (Falah et al. 2010)) to guide and stimulate the formation of cartilage tissue (Seo et al. 2014). During microfracture surgery an arthroscopic awl is used to penetrate the subchondral bone, while with subchondral drilling a high speed drill is applied to penetrate the trabecular bone. Different strategies have been applied including the implantation of biomaterials with and without cells. 
84 Acellular biomaterials offer various advantageous properties such as lack of donor-site 85 morbidity, absence of cell culture costs, off the shelf availability, fewer regulatory issues, and application of one-stage surgical procedures (Brouwer et al. 2011; Efe et al. 2012). Many researchers have explored the approach of implanting acellular biomaterials and investigated the use of various biomaterials in vivo, such as natural (e.g. collagen (Breinan et al. 2000; Buma et al. 2003; Enea et al. 2013; Wakitani et al. 1994), chitosan (Abarrategi et al. 2010; Bell et al. 2013; Guzman-Morales et al. 2014; Hoemann et al. 2007), alginate (Igarashi et al. 2012; Mierisch et al. 2002; Sukegawa et al. 2012) and hyaluronic acid (Aulin et al. 2013; Kayakabe et al. 2006; Marmotti et al. 2012; Solchaga et al. 2000)) and synthetic polymers (e.g. polycaprolactone (Christensen et al. 2012; Martinez-Diaz et al. 2010; Mrosek et al. 2010), polyvinyl alcohol (Coburn et al. 2012; Holmes et al. 1975; Krych et al. 2013) and poly(lactic-co-glycolic acid) (Athanasiou et al. 1997; Chang et al. 2012; Cui et al. 2009; Fonseca et al. 2014)). To combine the advantageous properties of these materials, multilayered biomaterials (e.g. $\beta$-tricalcium phosphate-hydroxyapatite/hyaluronate-atelocollagen (Ahn et al. 2009), ceramic bovine bonegelatin/gelatin-chondroitin sulfate-sodium hyaluronate (Deng et al. 2012)), blends (e.g. poly(glycolic acid)-hyaluronic acid (Erggelet et al. 2009) and type I collagen-hyaluronic acidfibrinogen hydrogel (Lee et al. 2012)) have been constructed. Biologics are natural factors that can be used to stimulate tissue regeneration, e.g. by inducing proliferation and differentiation of cells. Biologics such as growth factors of the transforming growth factor $\beta$ (TGF- $\beta$ ) superfamily and others have been incorporated in biomaterials to guide and stimulate the formation of hyaline cartilage tissue (Richter 2009). Moreover, it has been reported that the animal model of choice may have a significant impact on study outcome of articular cartilage regeneration (Reinholz et al. 2004). Currently, there is no systematic overview of the current literature assessing the effect of various parameters (e.g. applied biomaterials, incorporated biologics and animal models) on cartilage regeneration.

The aim of this systematic review and meta-analysis is to assess all current evidence for the efficacy of articular cartilage regeneration using acellular biomaterials implanted in the knee and ankle joint after microfracture and subchondral drilling in animal models. Additionally, we

112 strive to provide transparency on the quality of performed in vivo studies, in order to aid the 113 design of future animal experiments and clinical trials. We provide a systematic and unbiased 114 overview of the current literature addressing regeneration of articular cartilage using a wide 
115 range of acellular biomaterials containing various biological cues (as illustrated in Fig. 1). Results

116 of semi-quantitative histological scoring systems are used as a quantitative outcome parameter

117 for outcome assessment of cartilage regeneration. Although microfracture surgery and

118 subchondral drilling strive to stimulate cartilage and osteochondral regeneration, respectively,

119 both are generalized in this study as cartilage regeneration. Moreover, the evaluation of

120 different subgroups (natural and synthetic origin of the biomaterials, structure of the materials

121 (scaffolds vs. hydrogels), incorporated biological cues, and animal models) was included to gain

122 insights in which parameters affect cartilage regeneration and to what extent.

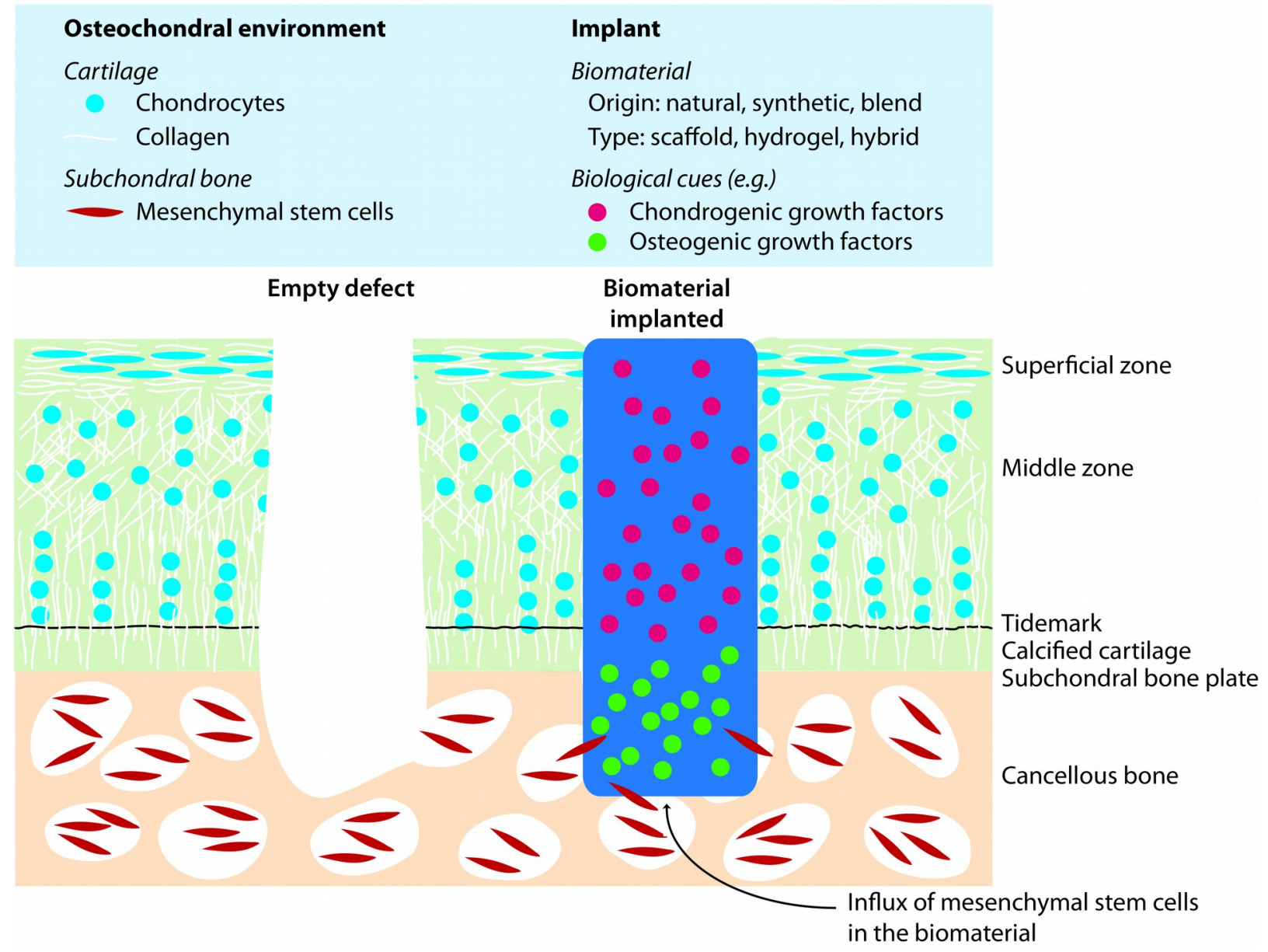

123 Figure 1. Illustration of cartilage regeneration by implantation of biomaterials after bone marrow

124 stimulation. The implanted biomaterials provide a template to guide cartilage regeneration by 125 bone marrow derived mesenchymal stem cells. 
126

127

128

130

132

\section{Materials and Methods}

\subsection{Search strategy}

To identify relevant peer-reviewed articles, a comprehensive search of the literature using PubMed and EMBASE (via OvidSP) was conducted, using the methods defined by de Vries et al. (de Vries et al. 2012) and Leenaars et al. (Leenaars et al. 2012). The last search date was April $3^{\text {rd }}$ 2015. In both databases, a tissue engineering search component developed by Sloff et al. (Sloff et al. 2014), consisting of equivalents for tissue engineering (e.g. tissue regeneration, regenerative medicine, bio-engineering or biomatrices), was combined with a cartilage search component, consisting of equivalents for cartilage and cartilage-related surgeries (e.g. chondral, chondrogenic, surgery, microfracturing or implants). The search components were constructed using MeSH terms (PubMed) and EMTREE terms (EMBASE) and additional free-text words from titles or abstracts ([tiab] or ti,ab). The obtained tissue engineering-related and cartilage-related results were filtered for animal studies using previously described animal search filters (de Vries et al. 2011; Hooijmans et al. 2010). The complete search strategy is attached in Supplementary Information 1. No language restrictions were used.

\subsection{Study selection}

References from the PubMed and EMBASE search strategies were combined and duplicates were manually removed from EndNote, with the preference of PubMed over EMBASE. All screening phases were performed by two independent reviewers (MP and VG) and reported according to the "Preferred Reporting Items for Systematic Reviews and Meta-Analysis" (PRISMA) guidelines (Higgins 2011). References were first screened based on title and were excluded based on the following criteria: 1) titles showed no relevance to regeneration of articular (hyaline) cartilage, 2) it was specifically stated in the title that the conducted experiment was an in vitro study only, 3) osteoarthritis animal models were used, 4) only ex vivo studies were performed, and 5) deceased animals were used. In case of doubt or disagreement, references were included for further screening. The second screening phase consisted of a title/abstract screening in Early Review Organizing Software (EROS, Institute of Clinical Effectiveness and Health Policy, Buenos Aires, Argentina, www.eros-systematic-review.org). References were included based on the following inclusion criteria: 1) primary study, 2) animal model, 3) bone marrow stimulation by microfracturing or creation of an osteochondral defect, 
156 and 4) biomaterial implantation. Articles were only excluded when it was specifically stated in 157 the abstract that the study was performed without healthy animals or acellular biomaterials, or 158 if biomaterials were not implanted in the knee or ankle joint. Articles were not excluded in case 159 important information in the abstract was missing. These articles were assessed in the full-text 160 screening phase. For the full-text screening, articles were included if they met all of the 161 following inclusion criteria: 1) primary study, 2) animal model, 3) healthy animals, 4) articular 162 cartilage regeneration, 5) knee or ankle joint, 6) bone marrow stimulation by microfracturing or 163 creation of an osteochondral defect, and 7) implantation of an acellular biomaterial. In general, 164 if results of the two reviewers were different, articles were discussed until consensus was 165 reached. In case of double publication, one of the studies was removed. During the screening 166 phase, no selection was made based on publication language. The risk of bias assessment and 167 meta-analysis was applied to studies with a comparison between a non-treated empty defect 168 control and biomaterial implantation, and with semi-quantitative histological scoring system results as outcome data.

\subsection{Study characteristics}

From the studies included after the full-text screening, the following details were obtained: general information (author and year of publication), animal characteristics (species, strain, sex, age, weight and the number of animals), information related to the surgical defect (size, depth and location), experimental conditions, biomaterial, biologics, evaluation time points and all outcome measures used, i.e. macroscopic evaluation, semi-quantitative macroscopic evaluation, histology, immunohistochemistry, semi-quantitative histological scoring, and biomechanical tests. Data from semi-quantitative histological scorings were used in the meta-analysis (described in section 2.5). Histological scoring systems applied in different studies consisted of scoring parameters like cell morphology, Safranin-O staining, integrity of surface, thickness, surface of area filled with cells, chondrocyte clustering, degenerative changes, restoration of the subchondral bone and integrity.

\subsection{Risk of bias assessment}

A risk of bias analysis was performed to assess the methodological quality of the studies included in the meta-analysis, using an adapted version of the risk of bias tool described by 
185 Hooijmans et al. (Hooijmans et al. 2014) (for all included studies containing a 'non-treated

186 empty defect' as control group and studies using semi-quantitative histological scoring systems

187 as outcome measure). A flowchart was constructed (Supplementary Information 2) to score for

188 selection, performance, detection and attrition bias, where the scores '-', '?' and '+' indicate a

189 low, unknown and high risk of bias, respectively. The questions addressed are specified in the

190 Supplementary Information 3. Articles were scored independently by MP and VG, and if the

191 results of the two reviewers were different, results were discussed until consensus was reached.

192 All articles written in Chinese (16 studies) were excluded from the risk of bias assessment only,

193 due to limited resources to independently translate these articles by two native Chinese

194 speakers. However, the data of these studies were extracted and used in the meta-analysis.

$195 \quad 1.5$ Analysis preparations and meta-analysis

196 2.5.1 Analysis preparations

197 The statistical analyses were restricted to those studies containing the outcome measure semi-

198 quantitative histology, making a comparison between a 'non-treated empty defect' as control

199 group and implanted biomaterials as experimental group. Data (mean, standard deviation (SD)

200 and number of animals) of the control and experimental group were extracted from the studies,

201 for all available time points. When results were not given numerically, but depicted graphically,

202 the mean and SD were measured using ImageJ (1.46r, National Institutes of Health USA). For

203 studies presenting results in boxplots, the mean and standard deviation were recalculated from

204 the median, range and the sample size according the method described by Hozo et al. (Hozo et

205 al. 2005). When data were described by a mean and confidence interval $(\mathrm{Cl})$, the $\mathrm{Cl}$ was

206 recalculated to a standard deviation by the following equation:

$$
\text { standard deviation }=\sqrt{N} \times \frac{\text { up per limit }- \text { lower limit }}{3.92} \text { for a 95\% Cl (Higgins 2011). For some }
$$

208 studies, data were unclear and assumptions were made, which are listed in Supplementary

209 Information 3a. To compare studies with different histological score system scales, means and

210 standard deviations were converted to a $100 \%$ scale by dividing the result by the maximum

211 achievable histological score and multiplying by $100 \%$. In case of missing or unclear data,

212 authors were e-mailed to retrieve the data. When data could not be obtained, these studies

213 were excluded from the meta-analysis (reasons for exclusion are also given in Supplementary 
214 Information 2). Results of studies with several experimental groups were combined, following

215 the approach described in the Cochrane Handbook, table 7.7 (Higgins 2011). The same approach

216 was followed to combine results of different animals on several time points in the same group in

217 the same study. One study (Hamanishi, 2013) had an SD of zero, which caused problems in the

218 analyses. Therefore, the SD was changed to 4.29, equal to the SD of the experimental group of

219 the same study at the same time point. The resulting data were used to calculate the treatment

220 effect and corresponding standard error (SE) per study.

\subsubsection{Meta-analysis}

The following main research question was assessed: Does an overall beneficial effect exist of implanting acellular biomaterials in osteochondral defects compared to non-treated empty defects?

First, in order to select the appropriate statistical random-effects meta-analysis model, we compared a univariate approach to the bivariate approach. In the bivariate approach, separate outcomes for control and experimental group were used with their respective SEs. The correlation between these two outcomes was modeled with a compound symmetry covariance matrix, as this resulted in a much lower Akaike Information Criterion value than the use of an unstructured covariance matrix. Results were compared with those of the univariate approach, based on the treatment effect and SE per study. Results of the univariate and bivariate approaches were very similar and we therefore proceeded with the univariate approach, when applicable in combination with likelihood ratio tests.

Restricted to the experimental groups, the following sub-questions were addressed to evaluate whether the treatment effect depended on specific variables: 1) Is there a difference between the use of natural and synthetic biomaterials?; 2) Does the structure of the biomaterials affect cartilage regeneration?; 3) Do differences among various material subgroups exist?; 4) Does incorporation of biologics have a beneficial effect on cartilage regeneration compared to control biomaterials?; 5) Do differences among subgroups of biologics exist?; 6) Do different animal models result in variations in cartilage regeneration? Results are shown as \% cartilage regeneration $(95 \% \mathrm{Cl}$ : [lower $\mathrm{Cl}$, upper $\mathrm{Cl}$ ]. Some studies have more than one experimental group. Therefore, the total number of studies and number of experimental groups (no. of studies/groups) are provided. 
244 Sensitivity analyses were performed to evaluate the effect of time (e.g. all time points, 245 short (<=8 weeks), long time points (>8 weeks), or the maximum time point), outliers (excluding 246 consecutively the studies with the 10\% highest / lowest pooled SD, and studies with the 10\% 247 highest / lowest SE), implant location, bone marrow stimulating technique applied 248 (microfracturing vs. subchondral drilling), language (excluding studies reported in Chinese as the 249 risk of bias of these studies was not assessed), and excluding studies where assumptions had to 250 be made. Based on a pilot analysis, data of all time points were used for subgroup analyses. 251 Subgroup analyses were only performed for subgroups consisting of more than two groups.

252 The statistical analyses were performed with SAS/STAT ${ }^{\circledast}$ software version 9.2 for 253 Windows, copyright (C) 2002-2008 by SAS Institute Inc., Cary, NC, USA. The funnel plot shows the 254 overall outcome of the pooled effect size of each study. $I^{2}$ was used as a measure of 255 heterogeneity. The forest plot was created with ReviewManager (RevMan, Version 5.3. 256 Copenhagen: The Nordic Cochrane Centre, The Cochrane Collaboration, 2014). 
2573 Results

\section{$258 \quad 1.6$ Search and study inclusion}

259 The searches conducted in PubMed and EMBASE (Supplementary Information 1) resulted in 2604401 and 5986 studies, respectively, leaving 6688 studies after removal of duplicates. These 261 studies were screened by title and title/abstract, which resulted in 1088 included studies after 262 the title screening and 517 included studies after the title/abstract screening. Screening articles 263 by full-text and subsequently selection for studies with empty defect controls as well as semi264 quantitative histology as outcome measure resulted in 283 included studies after full-text 265 assessment, of which 151 and 135 articles could be used for the meta-analysis and risk of bias 266 assessment, respectively (Fig. 2). The studies from Xie et al. (Xie et al. 2014), Yao et al. (Yao et al. 267 2000) and Zhou et al. (Zhou \& Yu 2014) could not be retrieved as a full text and these studies 268 were therefore excluded. An overview of all included studies after full-text assessment as well as 269 studies included for the risk of bias assessment and meta-analysis is provided in Supplementary 270 Information 3a. All references and abbreviations can be found in Supplementary Information 3b. 271 In this table, remarks are provided related to exclusion reasons for risk of bias assessment and 272 meta-analysis (e.g. duplicate publication and incomplete data). Assumptions made for certain 273 studies are also stated in this table. 


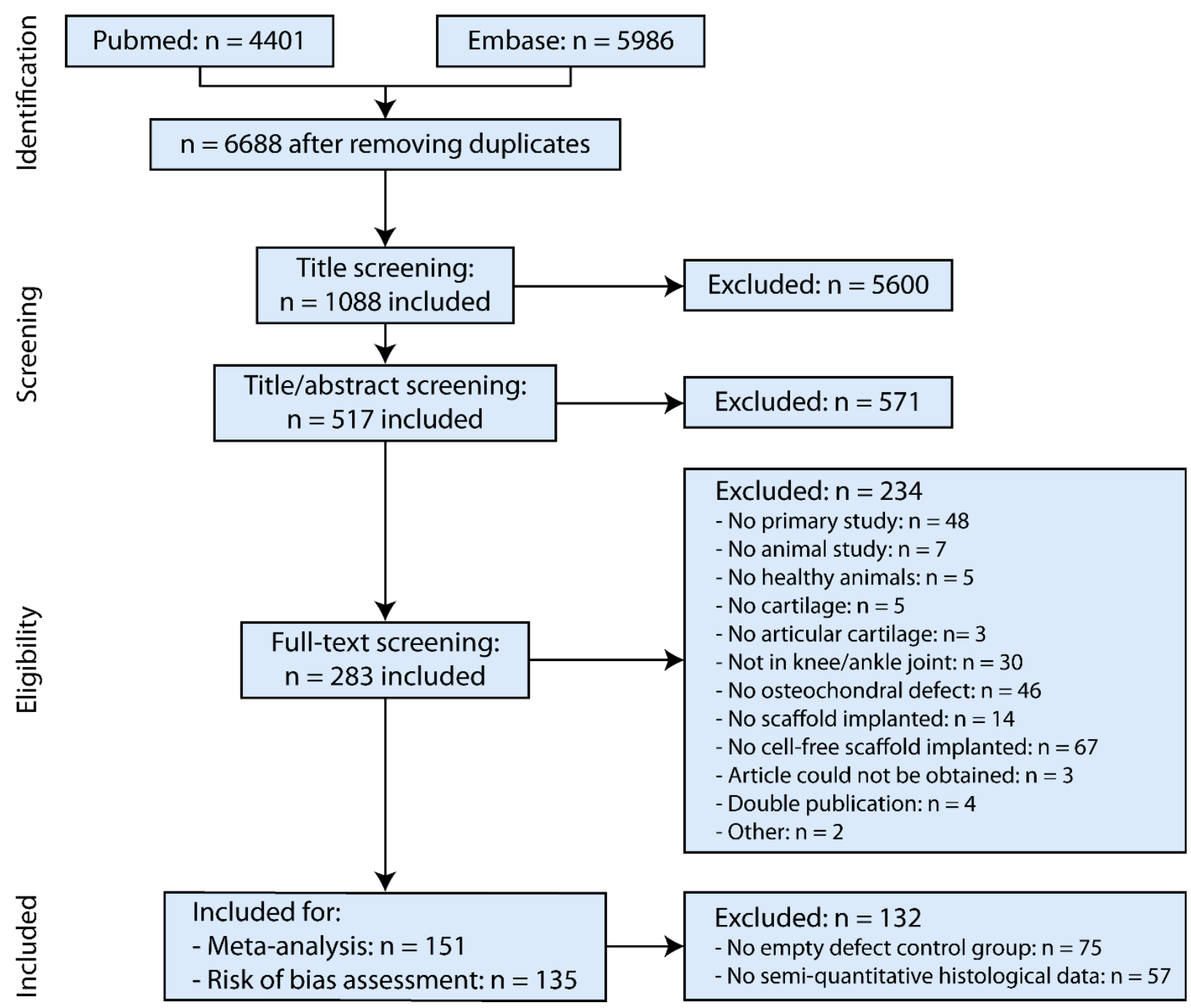

274 Figure 2. PRISMA (Preferred Reporting Items for Systematic Reviews and Meta-analysis)

275 flowchart of the systematic search of literature.

\subsection{Study characteristics}

277 The study characteristics (Supplementary Information 3) clearly show substantial variation

278 among studies. A wide range of animal species was used, from small (rat and rabbit) to larger

279 animal models (dog, minipig, goat, pig, sheep and horse). A large variation was observed

280 between the ages of animals (e.g. the age of rabbits ranged from 6 weeks to $>2$ years). Often

281 ages were not described or specified specifically (e.g. as adult or mature). Generally, the animals

282 were older (range of years) in large animal models compared to animals used in small animal

283 models (range of months). The defects were created at different locations in the knee joint, such

284 as the trochlea, condyle (medial and lateral), femur and intercondylar fossa. In addition, a large

285 variation was found in the dimensions of the prepared defects, e.g. the dimensions of the 
286 defects created in rabbits ranged from 4-7 $\mathrm{mm}$ in diameter and 0.8-9 $\mathrm{mm}$ in depth. 287 Microfracture surgery and subchondral drilling was performed in 25 and 258 studies, 288 respectively. The implanted biomaterials were of natural or synthetic origin or combinations 289 thereof, and consisted of single-layered or multilayered implants or blends thereof. Implants 290 were constructed from a wide range of materials or combinations thereof, such as collagen, 291 chitosan, hyaluronic acid, alginate, fibrin, hydroxyapatite, poly(lactic-co-glycolic acid), 292 polycaprolactone, poly(glycolic acid) and poly(ethylene glycol), and used in different states: 293 scaffolds, hydrogels, or hybrid mixtures of both. Various biological cues were incorporated in the 294 biomaterials prior to implantation or administered afterwards by injection into the knee joint, mostly growth factors of the TGF- $\beta$ superfamily such as bone morphogenetic protein 2 (BMP-2) and TGF- $\beta 1$, but also fibroblast growth factor (FGF) and platelet-rich plasma (PRP). The maximum follow-up time was 1 year, but studies mainly investigated relatively short-term effects of implanted biomaterials on cartilage regeneration (up to 6 months).

\subsection{Risk of bias assessment}

300 A risk of bias assessment was performed to assess risks of bias (selection, performance bias, detection and attrition bias) in studies included for the meta-analysis (Figure 3). An overview of all scores per individual study is provided in Supplementary Information 4.

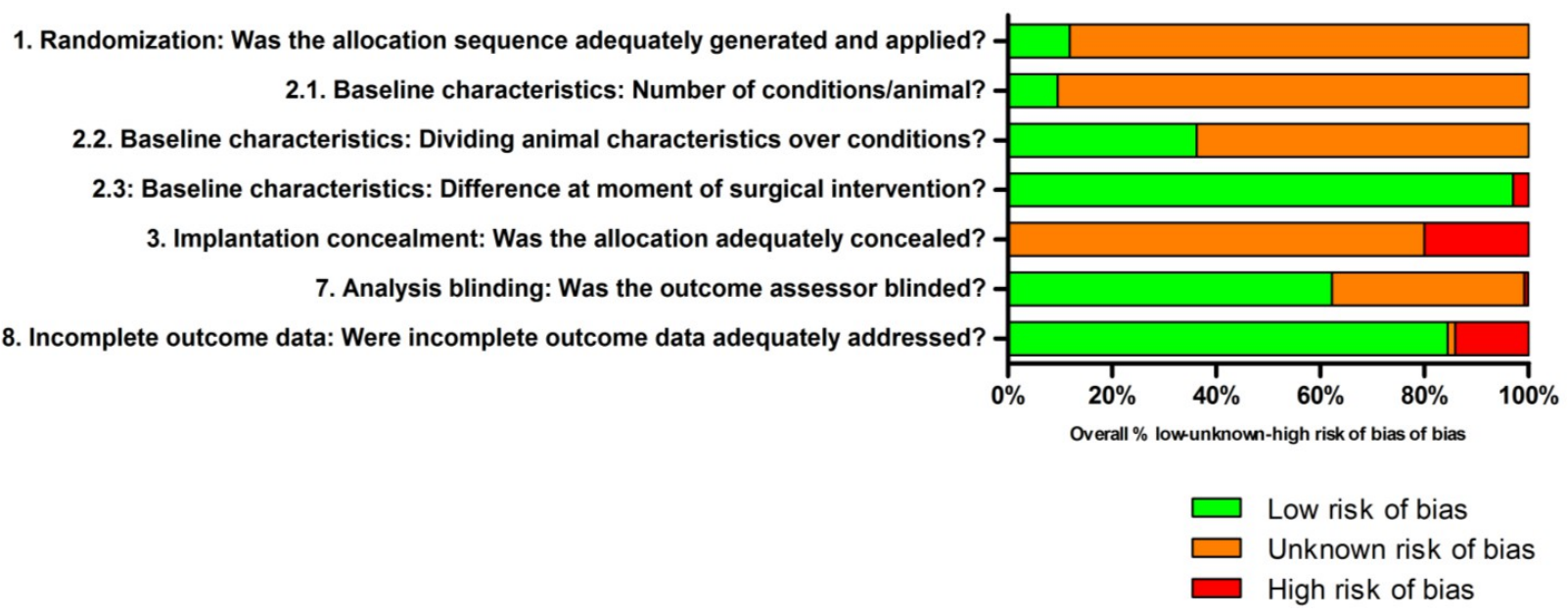

Figure 3. Risk of bias of all included studies in the meta-analysis. The green, orange and red colors depict the percentages of studies with low, unknown or high risk of bias of the total number of assessed studies. The risk of bias assessment indicated a general lack of details regarding the experimental setup, as indicated by the orange bars. The green bars represent a 
307 low risk of bias, mainly for the difference between groups at the moment of surgical intervention

308 and addressing incomplete outcome data. High risk of bias was infrequently scored, as indicated

309 by the red bars. Q4-Q6 are not depicted in the graph, but are described in Supplementary 310 Information 4.

311 The risk of bias assessment showed that details with respect to the randomization method were 312 not provided (Q1). It was often described that animals were randomized across different groups 313 without describing the method of randomization, thereby limiting assessment of the adequacy 314 of randomization and therefore the actual risk of selection bias. Another notable observation 315 from the experimental designs studied was that only in a limited number of studies it was 316 described that power calculations were performed, whereas sufficient power in animal 317 experiments is a requirement for performing adequate studies. The actual power analyses were never provided in the studies. Due to a lack of information, it was also difficult to assess possible bias by differences in implantation sites (with differences in load-bearing conditions, Q2.1) and differences between groups related to the age, sex and weight of the animals at the start of the experiment (Q2.2). Generally, baseline characteristics of animals prior to implantation of biomaterials (e.g. some animals received additional surgery related to harvesting of cells for biomaterials combined with cells, Q2.3) were similar. When implanting biomaterials, no details were described on blinding different biomaterials (Q3). Blinding of the empty defect and biomaterial conditions should be performed to limit bias. However, blinding between the empty defect and biomaterial group is impossible in case only one biomaterial is implanted. More than half of the studies conducted blinded outcome assessment while performing the histological scoring, resulting in low risk of detection bias, whereas the other studies had an unknown risk (Q7). For most studies, no incomplete outcome data were described/found, resulting in low risk of attrition bias. For some studies, dropouts were described/found, resulting in differences between groups and high risk of bias (Q8). Overall, the risk of bias analysis generally revealed

332 poor reporting of the experimental design for the majority of the studies, impeding an 333 assessment of the actual risk of bias. 


\subsection{Data synthesis}

335 For an overview of the meta-analysis and results obtained, see Table 1. The histological scores of

336 defects implanted with biomaterials and non-treated empty defects are presented as a 337 percentage on a $100 \%$ scale, where $0 \%$ and $100 \%$ indicate poor and perfect cartilage 338 regeneration, respectively. Data are presented as the effect (\%) with $95 \% \mathrm{Cl}$.

\begin{tabular}{|c|c|c|c|c|}
\hline Meta-analysis & $\begin{array}{c}\text { No. of } \\
\text { studies/groups }\end{array}$ & Subgroups & Cartilage regeneration $(\%[95 \% \mathrm{CI})]$ & $\begin{array}{l}\text { Mean difference (\% }[95 \% \mathrm{Cl}]) \\
\text { p-value }\end{array}$ \\
\hline \multirow{2}{*}{ 1. Overall effect } & $127 / 400$ & Biomaterial & $53.6[50.7,56.6]$ & $15.6[12.6,18.6]$ \\
\hline & $127 / 247$ & Empty defect & $38.1[35.1,41.0]$ & $\mathrm{p}<0.0001$ \\
\hline \multirow{2}{*}{ 2. Origin materials } & $76 / 222$ & Natural & $53.0[49.3,56.6]$ & $-0.73[-6.5,5.0]$ \\
\hline & $39 / 137$ & Synthetic & $53.7[48.8,58.7]$ & $p=0.887$ \\
\hline \multirow{8}{*}{ 3. Material subgroups } & $20 / 68$ & Collagen & $49.5[41.1,57.8]$ & \multirow{8}{*}{$p=0.804$} \\
\hline & $6 / 17$ & Chitosan & $57.5[40.8,74.2]$ & \\
\hline & $5 / 11$ & Hyaluronic acid & $47.9[31.7,64.1]$ & \\
\hline & $5 / 16$ & Alginate & $63.0[46.9,79.00]$ & \\
\hline & $3 / 10$ & Fibrin & $55.3[34.4,76.3]$ & \\
\hline & $5 / 11$ & Bone & $51.2[35.2,67.2]$ & \\
\hline & $15 / 52$ & PLGA & $58.5[49.0,68.0]$ & \\
\hline & $6 / 21$ & PAMPS-PDMAAm DN & $47.9[31.7,64.1]$ & \\
\hline \multirow{3}{*}{ 4. Scaffold structure } & $78 / 258$ & Scaffolds & $53.1[49.5,56.7]$ & \multirow{3}{*}{$p=0.973$} \\
\hline & $41 / 127$ & Hydrogels & $54.2[49.4,59.1]$ & \\
\hline & $7 / 17$ & Blends & $55.7[42.0,69.3]$ & \\
\hline \multirow{2}{*}{ 5. Biologicals } & $113 / 291$ & No biologicals & $51.7[48.6,54.9]$ & \multirow{2}{*}{$\begin{array}{c}7.56[2.1,13.0] \\
p=0.007\end{array}$} \\
\hline & $35 / 109$ & Biologicals & $59.3[54.0,64.6]$ & \\
\hline \multirow{4}{*}{ 6. Biological cues } & $9 / 35$ & BMP & $56.6[-6.3,119.6]$ & \multirow{4}{*}{$p=0.780$} \\
\hline & $5 / 20$ & FGF & $51.8[-43.9,147.4]$ & \\
\hline & $8 / 14$ & PRP & $55.9[-20.9,132.8]$ & \\
\hline & $6 / 16$ & TGF & $60.2[-7.5,128.0]$ & \\
\hline \multirow{7}{*}{ 7. Animal models } & $3 / 5$ & Dogs & ED: $31.9[14.5,49.4] ;$ B: $50.6[33.0,68.2]$ & $18.7[-0.0,37.3]$ \\
\hline & $5 / 13$ & Goats & ED: 58.5 [43.4, 73.7]; B: $61.6[47.6,75.6]$ & $3.1[-13.2,19.4]$ \\
\hline & $1 / 3$ & Macaques & ED: $12.2[-18.2,42.6] ;$ B: $6.8[-23.3,37.0]$ & $-5.4[-37.6,26.8]$ \\
\hline & $10 / 20$ & Minipigs & ED: $42.4[32.4,52.4] ;$ B: $56.1[46.3,66.0]$ & $13.6[3.1,24.1]$ \\
\hline & $94 / 333$ & Rabbits & ED: $37.7[34.2,41.1] ;$ B: $52.5[49.0,55.9]$ & $14.8[11.1,18.5]$ \\
\hline & $13 / 23$ & Sheep & ED: $35.3[26.4,44.3] ;$ B: $61.3[52.7,70.0]$ & $26.0[16.3,35.7]$ \\
\hline & & & & $p=0.348$ \\
\hline
\end{tabular}

339 Table 1. Overview of the meta-analysis results for the main research question assessing the

340 overall beneficial effect of implanting acellular biomaterials in osteochondral defects compared

341 to non-treated empty defects and sub-questions evaluating the effect of specific variables on the

342 treatment effect. The total number of studies and number of experimental groups included in 343 the meta-analysis are shown (some studies have $>1$ experimental group, no. of studies/groups).

344 The quality of cartilage regeneration is presented on a $100 \%$ scale, where $100 \%$ represents the 
345 maximum achievable histological score and thus the best cartilage regeneration. Implantation of

346 biomaterials significantly improved cartilage regeneration compared to non-treated empty 347 defects, which was further improved by the incorporation of biologics. No significant differences

348 were found between natural and synthetic materials, between the various material subgroups, 349 and between the biomaterial structures (hydrogels versus scaffolds versus blends), and between 350 animal species. ED: empty defect, B: biomaterials.

\subsubsection{Overall effect biomaterial implantation}

352 The meta-analysis indicates a significant improvement of cartilage regeneration using acellular 353 biomaterials implanted after applying marrow stimulating techniques compared to non-treated 354 empty defects (15.6\% (95\% Cl: [12.6, 18.6], $\mathrm{p}<0.0001)$. The forest plot (Supplementary 355 Information 5) depicts the outcome effect of each individual study. In 73 studies cartilage 356 regeneration significantly improved by the incorporation of biomaterials. In 48 studies no effect 357 was found, whereas in only 6 studies a negative effect on cartilage regeneration was observed. A 358 similar significant effect was observed taking into account the maximum follow-up only (16.3\% 359 [13.1, 19.6], $\mathrm{p}<0.0001)$. Also for short and long term follow-up cartilage regeneration was 360 significantly improved ( $\leq 8$ weeks: $12.5 \%$ [9.3, 15.7], > 8 weeks: 17.1\% [13.9, 20.2]). No notable 361 differences in cartilage regeneration were found between the results based on the maximum 362 follow-up time per study versus those based on all time points per study. Therefore, further 363 subgroup analyses were made using results from all time points together.

\subsubsection{Natural and synthetic materials}

The subgroup analysis assessing cartilage regeneration using materials of different origin, natural and synthetic, indicated no significant differences $(p=0.887)$ between natural $(53.0 \%$ [49.31, 56.63]) and synthetic materials (53.7\% [48.75, 58.65]).

368 Dividing the group of materials into subgroups allows comparison of cartilage regeneration using different biomaterials. The following subgroups were studied: 1) collagen, 2) chitosan, 3) hyaluronic acid-based biomaterials), 4) alginate, 5) fibrin), 6) bone material-based, biomaterial subgroups were found (Table 1). 
373

374 Materials were divided in three groups based on their structure: 1) scaffolds, 2) hydrogels, and

375 3) blends. Cartilage regeneration was similar after use of scaffolds $(53.1 \%$ [49.53, 56.74]), 376 hydrogels $(54.2 \%[49.39,59.07])$ and blends $(55.7 \%[42.0,69.3), p=0.973$.

\section{$377 \quad 3.1 .4 \quad$ Biologics}

378 Incorporation of biologics in the biomaterials resulted in a statistically significant improvement in cartilage regeneration of $7.6 \%[2.1,13.0], p=0.007$, compared to the implantation of control biomaterials. Including only those studies with a direct comparison between control biomaterials and biomaterials loaded with biologics resulted in an improved cartilage regeneration of $14.6 \%[5.9,23.4], p=0.003$. Comparing various biological cues including BMP, FGF, PRP and TGF indicated no significant differences in improvement of cartilage regeneration between these biologics.

\subsubsection{Animal models}

Evaluation of the animal models used showed no significant differences $(p=0.348)$ between the effects of biomaterials implanted in dogs, goats, macaques, minipigs, pigs, rabbits, rats or sheep (Table 1).

\subsubsection{Sensitivity analyses}

Sensitivity analyses were performed to assess the robustness of the meta-analysis with respect to the overall effect. The sensitivity analyses indicated that exclusion of studies with assumptions and studies written in Chinese (no risk of bias assessment analyzed) had no effect on the estimated difference in biomaterial regeneration. Moreover, including only studies with SDs or SEs in the $10-90 \%$ range did not notably change of the overall outcome effect. In a posthoc analysis, we investigated cartilage regeneration using biomaterials implanted at different locations including condyles, femur, intercondylar fossa and the trochlea. No differences were found comparing these implant sites $(p=0.143)$. In another post-hoc analysis, we compared cartilage regeneration of empty defects or defects filled with biomaterials after applying microfracturing or subchondral drilling. For empty defects $(p=0.152)$ and biomaterial implants $(p=0.063)$ no significant differences between the two bone marrow stimulating techniques were found. 
$402 \quad 3.1 .7 \quad$ Publication bias

403 A funnel plot (Figure 4) was prepared for all included studies to analyze the overall comparison 404 between acellular biomaterials and non-treated empty defect controls. No extensive asymmetry 405 was observed, indicating an absence of considerable publication bias.

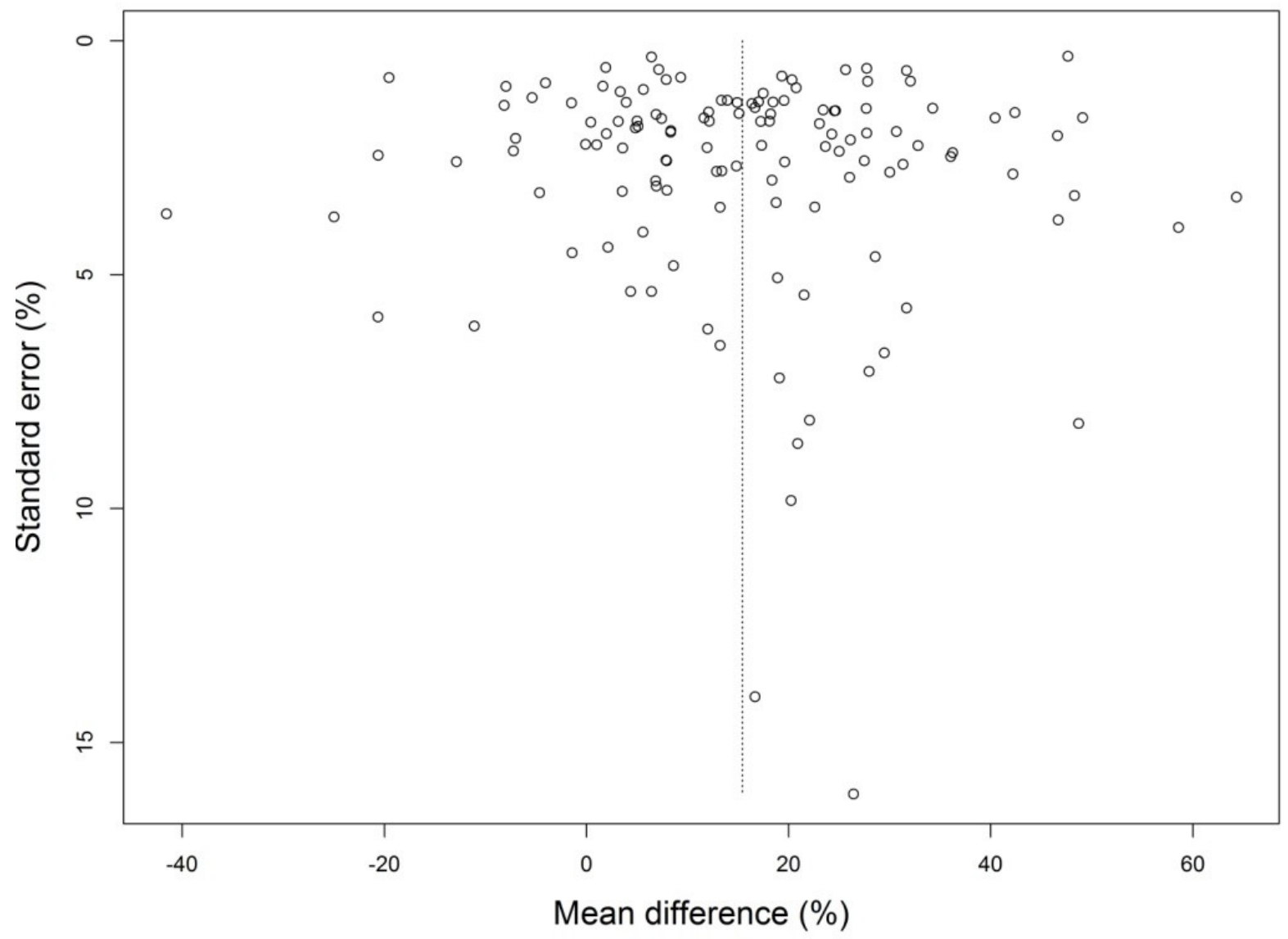

406 Figure 4. Funnel plot of included studies to assess the overall effect of the implantation of 407 acellular biomaterials compared to non-treated empty defect controls. The figure indicates no 408 substantial asymmetry. 
409

410

411

412

413

414

415

416

417

418

419

420

421

422

\section{Discussion}

The regeneration of damaged cartilage has been widely investigated using preclinical models. However, the efficacy of cartilage regeneration using implantation of acellular biomaterials has never been assessed using a systematic review and meta-analysis. This systematic review aimed (a) to provide an overview of currently existing knowledge and identify knowledge gaps, (b) to provide transparency on the quality of performed in vivo studies, and (c) to aid the design of future animal studies and clinical trials. The results could provide insight in strategies for future (pre) clinical research related to biomaterial properties, incorporation of biologics, choice of a suitable animal model, and their effects on cartilage regeneration.

The general findings of this systematic review and meta-analysis are that the implantation of biomaterials improves cartilage regeneration compared to non-treated osteochondral defects by $16 \%(95 \% \mathrm{Cl})$. There were only six out of 151 studies that showed a negative effect of biomaterial implantation on cartilage regeneration. In 48 studies no significant effect on cartilage regeneration was found. For those studies with improved cartilage regeneration (73 studies), clinical studies will have to confirm the beneficial effect of implantation of biomaterials on cartilage regeneration in human patients. Filardo et al. described the implantation of an osteochondral biomimetic scaffold consisting of a type I collagen cartilage-like layer, a type I collagen/hydroxyapatite intermediate layer, and a mineralized blend of type I collagen and hydroxyapatite as a subchondral bone compartment, to treat patients with osteochondritis dissecans. For these patients, clinical scores improved significantly after the first two years and evaluation by MRI indicated good defect filling and implant integration, but also heterogeneous tissue regeneration and changes of the subchondral bone (Filardo et al. 2013). In two studies included in this systematic review and meta-analysis, this osteochondral biomimetic scaffold was also implanted in sheep. Cartilage regeneration after six months was $81.8 \% \pm 8.9 \%$ (empty defect: $23.2 \% \pm 20.7 \%$ ) and $81.2 \% \pm 5.1 \%$ (empty defect: $23.4 \% \pm 6.7 \%$ ). A direct comparison between the degree of cartilage regeneration described in the preclinical studies and clinical study is not possible since no histological results were described in the clinical study. In addition, outcome measures used in preclinical studies may not predict the clinical outcome. For example, a randomized controlled clinical trial with BST-CarGel, a chitosan-based medical device, showed greater lesion filling and superior repair tissue quality compared to bone marrow stimulation after twelve months implantation, but without notable clinical differences related to pain, 
440 stiffness and physical function between both groups (Stanish et al. 2013). A remarkable 441 observation is the difference in follow-up between the studies, which may explain the good 442 histological scores in the preclinical studies after six months and heterogeneous tissue 443 regeneration and changes of the subchondral bone after two years in human patients. In 444 general, clinical studies demonstrated improved cartilage regeneration by the implantation of 445 biomaterials after bone marrow stimulation, but there is still room for improvement regarding 446 clinical outcome and tissue quality.

447 The only subgroup analysis that showed a statistically significant result between the 448 groups was between control biomaterials and biomaterials loaded with biologics. In future 449 clinical studies assessment of the beneficial properties of implanting biomaterials loaded with 450 biologics is of interest, since a significant improvement of $8 \%(95 \% \mathrm{Cl})$ compared to control 451 biomaterials was found and even $14.6 \%$ when using studies that directly compared biomaterials 452 with and without biologics. We were not able to perform analyses for the effect of the 453 concentration or subtype of the growth factors due to the small size of these subgroups, 454 although these factors may have a large effect on the outcome. In the study by Ishii et al. a 455 positive effect of FGF-2 was observed by the addition of at least $183 \mathrm{ng}$ to the biomaterials (Ishii 456 et al. 2007), while Maehara et al. showed significant improvements of impregnating of 457 biomaterials in $10 \mu \mathrm{g} / \mathrm{ml}$ and not for $100 \mu \mathrm{g} / \mathrm{ml}$ FGF-2 (Maehara et al. 2010). Loading 458 biomaterials with different BMPs including BMP-2 (Aulin et al. 2013; Reyes et al. 2012; Reyes et 459 al. 2014; Reyes et al. 2013; Tamai et al. 2005) and BMP-7 (Mori et al. 2013), or TGF subtypes 460 including TGF- $\beta$ (Mierisch et al. 2002) and TGF- $\beta 1$ (Reyes et al. 2012; Reyes et al. 2014), resulted 461 in significantly improved cartilage regeneration. However, for clinical application of these 462 medical devices, one should take safety of the products into account as side effects of TGF- $\beta$ in a 463 joint environment, including fibrosis and osteophyte formation, have been described (Blaney 464 Davidson et al. 2007) and patients suffered from major complications after spinal surgery and implantation of high concentrations of BMP/INFUSE (Epstein 2013).

466 The study characteristics of all included studies were tabulated to provide an extensive 467 overview of the available literature. Besides the internal validity of the studies, the 468 generalizability (external validity) of the study results is of great importance. The latter is 469 affected by factors related to the animal model (species, strain, weight, age, and sex), surgery 470 (location and size of the defect) and follow-up, resulting in heterogeneity between studies. This 
471 was also indicated by the relatively high level of heterogeneity $\left(\mathrm{I}^{2}\right)$ for the main meta-analysis $472(99.4 \%[99.4,99.4])$, and the heterogeneity was almost similar for subgroup analyses. We chose 473 to include only healthy animals receiving biomaterials. The screened studies also contained 474 osteoarthritis models that were not included, which may be relevant for future applications to 475 treat patients with osteoarthritis. Therefore, results from this systematic review and meta476 analysis may be different compared to results found for osteoarthritis models and future clinical 477 studies with osteoarthritis patients. We assumed that in order to assess the effect of implanted 478 biomaterials on cartilage regeneration, reduction of the influence of confounding parameters would aid the validity of the results and conclusions. In this study, the meta-analysis included all available data of the effect of implanting biomaterials after applying bone marrow stimulating techniques (microfracture and subchondral drilling) compared to empty defects on cartilage regeneration. During microfracture surgery the subchondral bone is penetrated using an arthroscopic awl, whereas during subchondral drilling the trabecular bone is penetrated using a high speed drill, which may result in thermal necrosis (Falah et al. 2010). Remarkably, more studies applied subchondral drilling (258 studies) compared to microfracture surgery (25 studies), while microfracture surgery was developed to overcome problems associated with thermal necrosis from subchondral drilling in the treatment of human patients (Kane et al. 2013). We did perform a post-hoc meta-analysis to investigate differences in cartilage regeneration after applying both marrow stimulating techniques and subsequent implantation of biomaterials, which resulted in no significant differences between microfracturing and subchondral drilling. A reason for the larger number of animal studies performing subchondral drilling compared to microfracture surgery may be the ease to perform subchondral drilling over microfracture surgery in animals. Although in the included studies, various implant locations (i.e. trochlea and condyles) were used, we grouped the results in the meta-analysis. A post-hoc subgroup analysis was performed to compare defect locations, but no overall significant differences were found for biomaterials implanted at different implant locations. Our analysis did not confirm a finding of Chen et al. showing improved chondrogenesis in trochlear versus condylar cartilage defects after bone marrow stimulation in rabbits (Chen et al. 2013). This may

499 be explained by various parameters affecting the degree of cartilage regeneration at different implant locations, such as the animal model, follow-up period and rehabilitation protocol. 
Different outcome measures such as macroscopic and histological evaluation, semiquantitative macroscopical and histological evaluation using scoring systems, histomorphometry, PCR and biochemical assays were used to assess the regenerative potential of implanting biomaterials. In this systematic review and meta-analysis, only data from semi-quantitative histological scoring systems were used as outcome measure. We chose to use these data as most authors presented their results by this method and it allows quantitative comparison of different studies in a meta-analysis. Various histological scoring systems have been used by the authors of included studies, such as the O'Driscoll, Pineda, Wakitani and ICRS scoring system, which were also reviewed by Rutgers et al. (Rutgers et al. 2010). Depending on the histological scoring system, parameters such as cell morphology, matrix staining, surface regularity, structural integrity, defect filling and the restoration of the subchondral bone were evaluated. A limitation of this outcome measure is that the specific topics addressed in the scoring systems greatly differ, i.e. some studies focus on the regeneration of cartilage only, cartilage as well as subchondral bone, or include a biomaterial component (e.g. scoring degradation of the implant). Other outcome measures including macroscopic evaluation, biochemical analysis and biomechanical aspects of the tissue may complete the overview of the tissue quality and provide valuable insights in articular cartilage regeneration, but these outcome measures were only used in a limited number of studies, and therefore not assessed in this analysis.

The risk of bias assessment provided insights in the quality of the experimental design of the studies. Most studies scored a low or unknown risk of bias, however, also little high risk of bias was scored. Low methodological quality (internal validity) may result in an overestimation or underestimation of the intervention effect (Higgins et al. 2011). In general, details regarding the randomization procedure were not described. Moreover, an observation during the risk of bias assessment was that only few studies included in the systematic review described that power calculations were performed, which is a crucial aspect in conducting experimental studies to ensure sufficient power of experimental designs. As a consequence, studies may lack sufficient power and thereby run the risk of false negative results. Due to the poor reporting of the experimental design for the majority of the studies the assessment of the adequacy of randomization and power calculations, and thus the assessment of the actual risk of selection bias, was inadequate. However, it may also hold true that studies were well designed but there was only poor reporting of the experimental designs (Hooijmans et al. 2012). Most researchers 
532 scoring the histology sections were blinded and sections were randomized. However, when

533 biomaterials are not (completely) degraded, blinding between biomaterials and empty defects is

534 practically impossible. A lack of blinding of outcome assessors implies the risk of

535 detection/observer bias (Bello et al. 2014). Bias may have been introduced by the lack of

536 blinding and randomization and detracts from the overall validity of the results (Bebarta et al.

537 2003; Hirst et al. 2014). There is a risk that the positive results found are an overestimation of

538 the true effect of using biomaterials. Introducing standardized protocols such as the golden

539 standard publication checklist (Hooijmans et al. 2011) or the ARRIVE guidelines (Kilkenny et al.

540 2012) may improve reporting of animal studies.

$541 \quad$ Funnel plots represent the precision of the measured effects, which increases by an

542 increase in study size. Therefore, for small and large studies scatter will be relatively large and

543 little, respectively. As a consequence, generally, in the absence of bias the plot resembles a

544 symmetrical pyramid (a funnel) (Higgins 2011). An important limitation may be publication bias,

545 since multiple studies were included from the same author and negative results may not be

546 published. It was described in a study by ter Riet et al. that researchers themselves estimate that

547 only $50 \%$ of the conducted animal experiments are published. This problem may be solved by

548 statistical corrections for publication bias (ter Riet et al. 2012). In our study, the funnel plot did

549 not show asymmetry and therefore did not indicate the presence of publication bias.

$550 \quad$ The translational value of animal studies depends on the comparability to the clinical

551 situation. One of the limitations of the performed animal experiments is the short follow-up

552 times. The maximum follow-up time was one year, but most studies investigated cartilage 553 regeneration up to 6 months. This limits the translational value since clinical improvements in 554 humans are generally observed up to 1.5 - 3 years after microfracture surgery (Hoemann et al. 555 2010; van der Linden et al. 2013). Moreover, many variations were present in the applied animal 556 models, i.e. animal characteristics (species, strain, sex, age, weight), surgical defects (size, depth 557 and location), applied biomaterials, and incorporated biologics. A review by Chu et al. (Chu et al. 558 2010) extensively reflects on benefits and limitations of different animal models used in cartilage 559 repair studies. They state that for humans the volume of a cartilage defect is approximately 550 $560 \mathrm{~mm}^{3}$ and treatment is required for defects with a surface larger than $10 \mathrm{~mm}^{2}$. Due to the limited 561 joint size of many animals, larger animal models such as minipig, goat and horse therefore offer 562 superior translational value than smaller animals such as rats, rabbits and dogs. However, all 
563 studies contained defect volumes smaller than $550 \mathrm{~mm}^{3}$ and only few studies had defects

564 surfaces larger than $10 \mathrm{~mm}^{2}$. Additionally, cartilage thickness differs among various species, with

565 goat, rabbit, minipig and dogs having thinner cartilage than humans. Another drawback for

566 some animal models is the large endogenous repair potential. In humans, untreated defects

567 show little to no regeneration while rabbits display a large regenerative potential, limiting clinical

568 translation. Dog, goat, minipig and horse do not have this large endogenous repair and the use

569 of these animals may therefore be favorable. The maturity of the animals is of great importance

570 when designing animal experiments since open growth plates can impede with the applied

571 treatment. Animal species are skeletally mature at different ages; i.e. rabbits at the age of 16-39

572 weeks, pigs at 42-52 weeks, dogs at 12-24 months, sheep and goat at 24-36 months and horses

573 at 60-72 months (Ahern et al. 2009; Chu et al. 2010). In this study we did not group studies

574 based on animal maturity. In addition to clinical relevance, other reasons to select an animal

575 model are related to logistical, financial, and ethical considerations. A systematic review

576 conducted by Ahern et al. (Ahern et al. 2009) investigated the strengths and shortcomings of

577 different animal models and compared these with common clinical lesions in clinical studies.

578 They remarked that smaller animal models are often used due to feasibility, while large animal

579 models may more closely resemble humans. However, no differences were found between

580 animal models in this systematic review and meta-analysis, which may be explained by various

581 parameters affecting the degree of cartilage regeneration such as implant location, defect size,

582 follow-up period and rehabilitation protocol.

583 In this systematic review and meta-analysis the efficacy of cartilage regeneration using

584 acellular biomaterials was compared to the natural healing response of defect treated with

585 microfracture surgery and subchondral drilling. The risk of bias assessment indicated poor

586 reporting in animal studies, which may be improved in future animal studies. Moreover, to

587 improve the translation towards clinical trials animal experiments should be comparable to the

588 clinical situation. As described in this systematic review a relatively high level of heterogeneity

589 exists between studies related to the animal model, surgery and follow-up, with a need to

590 resemble current clinical settings more closely. In this study we only addressed bone marrow

591 stimulating techniques (microfracture and subchondral drilling) and subsequently the

592 incorporation of biomaterials, but also the regeneration of partial thickness cartilage defects

593 may be beneficial to prevent progression to full-thickness cartilage defects, limit the progression 
594 towards osteoarthritis and improve quality of life in patients. In many studies also cell-laden 595 biomaterials have been implanted and the beneficial effect of cellular biomaterials versus 596 acellular biomaterials and the natural healing response has been studied. Although acellular 597 biomaterials offer various advantageous properties over cellular biomaterials such as no donor598 site, no cell culture, off the shelf availability, less regulatory issues, and application of one-stage 599 surgical procedures (Brouwer et al. 2011; Efe et al. 2012), studying the additive value of cellular 600 biomaterials may aid further improvement of marrow stimulating techniques. 
6015 Conclusion

602 The systematic review and meta-analysis resulted in a structured, thorough and transparent 603 overview of literature related to the current evidence for the efficacy of cartilage regeneration 604 using acellular biomaterials implanted after microfracturing in animal models. Cartilage 605 regeneration is more effective by implantation of acellular biomaterials in microfracture defects 606 compared to microfracturing alone. The efficacy is further improved by the incorporation of 607 biologics.

6086 Manuscript information and declarations

609 Acknowledgements:

610 We thank Jie An (Department of Biomaterials, Radboud Institute for Molecular Life Sciences, 611 Radboud university medical center) for full-text screening articles written in Chinese. Gerrie 612 Hermkens from the Radboud university medical center medical library is greatly acknowledged 613 for help retrieving full text studies. 
614

615

616

617

618

619

620

621

622

623

624

625

626

627

628

629

630

631

632

633

634

635

636

637

638

639

640

641

642

643

644

645

646

647

648

649

650

651

652

653

654

655

656

657

658

\section{References}

Abarrategi A, Lopiz-Morales Y, Ramos V, Civantos A, Lopez-Duran L, Marco F, and Lopez-Lacomba JL. 2010. Chitosan scaffolds for osteochondral tissue regeneration. Journal of Biomedical Materials Research Part A 95:1132-1141. 10.1002/jbm.a.32912

Ahern BJ, Parvizi J, Boston R, and Schaer TP. 2009. Preclinical animal models in single site cartilage defect testing: a systematic review. Osteoarthritis Cartilage 17:705-713. 10.1016/j.joca.2008.11.008

Ahn JH, Lee TH, Oh JS, Kim SY, Kim HJ, Park IK, Choi BS, and Im GI. 2009. Novel hyaluronateatelocollagen/beta-TCP-hydroxyapatite biphasic scaffold for the repair of osteochondral defects in rabbits. Tissue Engineering Part A 15:2595-2604. 10.1089/ten.TEA.2008.0511

Athanasiou K, Korvick D, and Schenck Jr R. 1997. Biodegradable implants for the treatment of osteochondral defects in a goat model. Tissue Engineering 3:363-373. 10.1089/ten.1997.3.363

Aulin C, Jensen-Waern M, Ekman S, Hagglund M, Engstrand T, Hilborn J, and Hedenqvist P. 2013. Cartilage repair of experimentally 11 induced osteochondral defects in new zealand white rabbits. Laboratory Animals 47:58-65. 10.1177/0023677212473716

Bal BS, Rahaman MN, Jayabalan P, Kuroki K, Cockrell MK, Yao JQ, and Cook JL. 2010. In vivo outcomes of tissue-engineered osteochondral grafts. Journal of Biomedical Materials Research Part B 93:164-174. 10.1002/jbm.b.31571

Bebarta V, Luyten D, and Heard K. 2003. Emergency medicine animal research: does use of randomization and blinding affect the results? Academic Emergency Medicine 10:14101410. 10.1111/j.1553-2712.2003.tb00056.x

Bell AD, Lascau-Coman V, Sun J, Chen G, Lowerison MW, Hurtig MB, and Hoemann CD. 2013. Bone-Induced Chondroinduction in Sheep Jamshidi Biopsy Defects with and without Treatment by Subchondral Chitosan-Blood Implant: 1-Day, 3-Week, and 3-Month Repair. Cartilage 4:131-143. 1177/1947603512463227

Bello S, Krogsboll LT, Gruber J, Zhao ZJ, Fischer D, and Hrobjartsson A. 2014. Lack of blinding of outcome assessors in animal model experiments implies risk of observer bias. Journal of Clinical Epidemiology 67:973-983. 10.1016/j.jclinepi.2014.04.008

Blaney Davidson EN, van der Kraan PM, and van den Berg WB. 2007. TGF-beta and osteoarthritis. Osteoarthritis Cartilage 15:597-604. 10.1016/j.joca.2007.02.005

Breinan HA, Martin SD, Hsu HP, and Spector M. 2000. Healing of canine articular cartilage defects treated with microfracture, a type-Il collagen matrix, or cultured autologous chondrocytes. Journal of Orthopaedic Research 18:781-789. 10.1002/jor.1100180516

Brouwer KM, van Rensch P, Harbers VE, Geutjes PJ, Koens MJ, Wijnen RM, Daamen WF, and van Kuppevelt TH. 2011. Evaluation of methods for the construction of collagenous scaffolds with a radial pore structure for tissue engineering. Journal of Tissue Engineering and Regenerative Medicine 5:501-504. 10.1002/term.397

Buma P, Pieper JS, van Tienen T, van Susante JL, van der Kraan PM, Veerkamp JH, van den Berg WB, Veth RP, and van Kuppevelt TH. 2003. Cross-linked type I and type II collagenous matrices for the repair of full-thickness articular cartilage defects--a study in rabbits. Biomaterials 24:3255-3263. S0142961203001431 [pii]

Cao Z, Hou S, Sun D, Wang X, and Tang J. 2012. Osteochondral regeneration by a bilayered construct in a cell-free or cell-based approach. Biotechnology Letters 34:1151-1157. 10.1007/s10529-012-0884-9 
659

660

661

662

663

664

665

666

667

668

669

670

671

672

673

674

675

676

677

678

679

680

681

682

683

684

685

686

687

688

689

690

691

692

693

694

695

696

697

698

699

700

701

702

703

704

Chang NJ, Lin CC, Li CF, Wang DA, Issariyaku N, and Yeh ML. 2012. The combined effects of continuous passive motion treatment and acellular PLGA implants on osteochondral regeneration in the rabbit. Biomaterials 33:3153-3163. 10.1016/j.biomaterials.2011.12.054

Chen H, Chevrier A, Hoemann CD, Sun J, Lascau-Coman V, and Buschmann MD. 2013. Bone marrow stimulation induces greater chondrogenesis in trochlear vs condylar cartilage defects in skeletally mature rabbits. Osteoarthritis Cartilage 21:999-1007. 10.1016/j.joca.2013.04.010

Christensen BB, Foldager CB, Hansen OM, Kristiansen AA, Le DQ, Nielsen AD, Nygaard JV, Bunger CE, and Lind M. 2012. A novel nano-structured porous polycaprolactone scaffold improves hyaline cartilage repair in a rabbit model compared to a collagen type I/III scaffold: in vitro and in vivo studies. Knee Surgery, Sports Traumatology, Arthroscopy 20:1192-1204. 10.1007/s00167-011-1692-9

Chu CR, Szczodry M, and Bruno S. 2010. Animal models for cartilage regeneration and repair. Tissue Engineering Part B 16:105-115. 10.1089/ten.TEB.2009.0452

Coburn JM, Gibson M, Monagle S, Patterson Z, and Elisseeff JH. 2012. Bioinspired nanofibers support chondrogenesis for articular cartilage repair. Proceedings of the National Academy of Sciences 109:10012-10017. 10.1073/pnas.1121605109

Cui YM, Wu J, and Hu YY. 2009. Repairing articular cartilage defects in rabbits using bone marrow stromal cell-derived chondrocytes compounded with poly(lactic-co-glycolic acid). Journal of Clinical Rehabilitative Tissue Engineering Research 13:10049-10054. 10.3969/j.issn.1673-8225.2009.51.009

Dai L, He Z, Zhang X, Hu X, Yuan L, Qiang M, Zhu J, Shao Z, Zhou C, and Ao Y. 2014. One-step repair for cartilage defects in a rabbit model: a technique combining the perforated decalcified cortical-cancellous bone matrix scaffold with microfracture. The American Journal of Sports Medicine 42:583-591. 10.1177/0363546513518415

de Mulder EL, Hannink G, van Kuppevelt TH, Daamen WF, and Buma P. 2014. Similar hyaline-like cartilage repair of osteochondral defects in rabbits using isotropic and anisotropic collagen scaffolds. Tissue Engineering Part A 20:635-645. 10.1089/ten.TEA.2013.0083

de Vries RB, Buma P, Leenaars M, Ritskes-Hoitinga M, and Gordijn B. 2012. Reducing the number of laboratory animals used in tissue engineering research by restricting the variety of animal models. Articular cartilage tissue engineering as a case study. Tissue Engineering Part B 18:427-435. 10.1089/ten.TEB.2012.0059

de Vries RB, Hooijmans CR, Tillema A, Leenaars M, and Ritskes-Hoitinga M. 2011. A search filter for increasing the retrieval of animal studies in Embase. Laboratory Animals 45:268-270. 10.1258/la.2011.011056

Deng T, Lv J, Pang J, Liu B, and Ke J. 2012. Construction of tissue-engineered osteochondral composites and repair of large joint defects in rabbit. Journal of Tissue Engineering and Regenerative Medicine 8:546-556. 10.1002/term.1556

Efe T, Theisen C, Fuchs-Winkelmann S, Stein T, Getgood A, Rominger MB, Paletta JR, and Schofer MD. 2012. Cell-free collagen type I matrix for repair of cartilage defects-clinical and magnetic resonance imaging results. Knee Surgery, Sports Traumatology, Arthroscopy 20:1915-1922. 10.1007/s00167-011-1777-5

Enea D, Guerra D, Roggiani J, Cecconi S, Manzotti S, Quaglino D, Pasquali-Ronchetti I, and Gigante A. 2013. Mixed type I and type II collagen scaffold for cartilage repair: Ultrastructural study of synovial membrane response and healing potential versus 
705

706

707

708

709

710

711

712

713

714

715

716

717

718

719

720

721

722

723

724

725

726

727

728

729

730

731

732

733

734

735

736

737

738

739

740

741

742

743

744

745

746

747

748

749

microfractures (a pilot study). International Journal of Immunopathology and Pharmacology 26:917-930. 10.1088/2058-7058/26/06/10

Epstein NE. 2013. Complications due to the use of BMP/INFUSE in spine surgery: The evidence continues to mount. Surgical Neurology International 4:S343-352. 10.4103/21527806.114813

Erggelet C, Endres M, Neumann K, Morawietz L, Ringe J, Haberstroh K, Sittinger M, and Kaps C. 2009. Formation of cartilage repair tissue in articular cartilage defects pretreated with microfracture and covered with cell-free polymer-based implants. Journal of Orthopaedic Research 27:1353-1360. 10.1002/jor.20879

Falah M, Nierenberg G, Soudry M, Hayden M, and Volpin G. 2010. Treatment of articular cartilage lesions of the knee. International Orthopaedics 34:621-630. 10.1007/s00264010-0959-y

Filardo G, Kon E, Di Martino A, Busacca M, Altadonna G, and Marcacci M. 2013. Treatment of knee osteochondritis dissecans with a cell-free biomimetic osteochondral scaffold: clinical and imaging evaluation at 2-year follow-up. The American Journal of Sports Medicine 41:1786-1793. 10.1177/0363546513490658

Fonseca C, Caminal M, Peris D, Barrachina J, Fabregas PJ, Garcia F, Cairo JJ, Godia F, Pla A, and Vives J. 2014. An arthroscopic approach for the treatment of osteochondral focal defects with cell-free and cell-loaded PLGA scaffolds in sheep. Cytotechnology 66:345-354. 10. 1007/s10616-013-9581-3

Guzman-Morales J, Lafantaisie-Favreau CH, Chen G, and Hoemann CD. 2014. Subchondral chitosan/blood implant-guided bone plate resorption and woven bone repair is coupled to hyaline cartilage regeneration from microdrill holes in aged rabbit knees. Osteoarthritis Cartilage 22:323-333. 10.1016/j.joca.2013.12.011

Higgins JP, Altman DG, Gotzsche PC, Juni P, Moher D, Oxman AD, Savovic J, Schulz KF, Weeks L, Sterne JA, Cochrane Bias Methods G, and Cochrane Statistical Methods G. 2011. The Cochrane Collaboration's tool for assessing risk of bias in randomised trials. BMJ 343:d5928. 10.1136/bmj.d5928

Higgins JPT, Green, S. 2011. Cochrane Handbook for Systematic Reviews of Interventions.

Hirst JA, Howick J, Aronson JK, Roberts N, Perera R, Koshiaris C, and Heneghan C. 2014. The need for randomization in animal trials: an overview of systematic reviews. PLoS One 9:e98856. 10.1371/journal.pone.0098856

Hoemann CD, Chen G, Marchand C, Tran-Khanh N, Thibault M, Chevrier A, Sun J, Shive MS, Fernandes MJ, Poubelle PE, Centola M, and El-Gabalawy H. 2010. Scaffold-guided subchondral bone repair: implication of neutrophils and alternatively activated arginase1+ macrophages. The American Journal of Sports Medicine 38:1845-1856. 10.1177/0363546510369547

Hoemann CD, Sun J, McKee MD, Chevrier A, Rossomacha E, Rivard GE, Hurtig M, and Buschmann MD. 2007. Chitosan-glycerol phosphate/blood implants elicit hyaline cartilage repair integrated with porous subchondral bone in microdrilled rabbit defects. Osteoarthritis Cartilage 15:78-89. 10.1016/j.joca.2006.06.015

Holmes M, Volz RG, and Chvapil M. 1975. Collagen sponge as a matrix for articular cartilage regeneration. Surgical Forum 26:511-513.

Hooijmans C, de Vries R, Leenaars M, and Ritskes-Hoitinga M. 2011. The Gold Standard Publication Checklist (GSPC) for improved design, reporting and scientific quality of 
Hooijmans CR, de Vries RB, Rovers MM, Gooszen HG, and Ritskes-Hoitinga M. 2012. The effects of probiotic supplementation on experimental acute pancreatitis: a systematic review and meta-analysis. PLoS One 7:e48811. 10.1371/journal.pone.0048811

Hooijmans CR, Rovers MM, de Vries RB, Leenaars M, Ritskes-Hoitinga M, and Langendam MW. 2014. SYRCLE's risk of bias tool for animal studies. BMC Medical Research Methodology 14:43. 10.1186/1471-2288-14-43

Hooijmans CR, Tillema A, Leenaars M, and Ritskes-Hoitinga M. 2010. Enhancing search efficiency by means of a search filter for finding all studies on animal experimentation in PubMed. Laboratory Animals 44:170-175. 10.1258/la.2010.009117

Hozo SP, Djulbegovic B, and Hozo I. 2005. Estimating the mean and variance from the median, range, and the size of a sample. BMC Medical Research Methodology 5:13. 10.1186/1471-2288-5-13

Igarashi T, Iwasaki N, Kawamura D, Kasahara Y, Tsukuda Y, Ohzawa N, Ito M, Izumisawa Y, and Minami A. 2012. Repair of articular cartilage defects with a novel injectable in situ forming material in a canine model. Journal of Biomedical Materials Research Part A 100:180-187. 10.1002/jbm.a.33248

Ishii I, Mizuta H, Sei A, Hirose J, Kudo S, and Hiraki Y. 2007. Healing of full-thickness defects of the articular cartilage in rabbits using fibroblast growth factor-2 and a fibrin sealant. The Journal of Bone and Joint Surgery 89:693-700. 10.1302/0301-620X.89B5.18450

Kane P, Frederick R, Tucker B, Dodson CC, Anderson JA, Ciccotti MG, and Freedman KB. 2013. Surgical restoration/repair of articular cartilage injuries in athletes. The Physician and Sportsmedicine 41:75-86. 10.3810/psm.2013.05.2017

Kayakabe M, Tsutsumi S, Watanabe H, Kato Y, and Takagishi K. 2006. Transplantation of autologous rabbit BM-derived mesenchymal stromal cells embedded in hyaluronic acid gel sponge into osteochondral defects of the knee. Cytotherapy 8:343-353. 10.1080/14653240600845070

Kilkenny C, Browne WJ, Cuthi I, Emerson M, and Altman DG. 2012. Improving bioscience research reporting: the ARRIVE guidelines for reporting animal research. PLoS Biology 41:27-31. 10.1111/j.1939-165X.2012.00418.x

Krych AJ, Wanivenhaus F, Ng KW, Doty S, Warren RF, and Maher SA. 2013. Matrix generation within a macroporous non-degradable implant for osteochondral defects is not enhanced with partial enzymatic digestion of the surrounding tissue: Evaluation in an in vivo rabbit model. Journal of Materials Science: Materials in Medicine 24:2429-2437. 1007/s10856013-4999-x

Lee JC, Lee SY, Min HJ, Han SA, Jang J, Lee S, Seong SC, and Lee MC. 2012. Synovium-derived mesenchymal stem cells encapsulated in a novel injectable gel can repair osteochondral defects in a rabbit model. Tissue Engineering Part A 18:2173-2186. 10.1089/ten.TEA.2011.0643

Leenaars M, Hooijmans CR, van Veggel N, ter Riet G, Leeflang M, Hooft L, van der Wilt GJ, Tillema A, and Ritskes-Hoitinga M. 2012. A step-by-step guide to systematically identify all relevant animal studies. Laboratory Animals 46:24-31. 10.1258/la.2011.011087

Maehara H, Sotome S, Yoshii T, Torigoe I, Kawasaki Y, Sugata Y, Yuasa M, Hirano M, Mochizuki N, Kikuchi M, Shinomiya K, and Okawa A. 2010. Repair of large osteochondral defects in 
795

796

rabbits using porous hydroxyapatite/collagen $(\mathrm{HAp} / \mathrm{Col})$ and fibroblast growth factor-2 (FGF-2). Journal of Orthopaedic Research 28:677-686. 10.1002/jor.21032

Marmotti A, Bruzzone M, Bonasia DE, Castoldi F, Rossi R, Piras L, Maiello A, Realmuto C, and Peretti GM. 2012. One-step osteochondral repair with cartilage fragments in a composite scaffold. Knee Surgery, Sports Traumatology, Arthroscopy 20:2590-2601. 10.1007/s00167-012-1920-y

Martinez-Diaz S, Garcia-Giralt N, Lebourg M, Gomez-Tejedor JA, Vila G, Caceres E, Benito P, Pradas MM, Nogues X, Ribelles JL, and Monllau JC. 2010. In vivo evaluation of 3dimensional polycaprolactone scaffolds for cartilage repair in rabbits. The American Journal of Sports Medicine 38:509-519. 10.1177/0363546509352448

Mierisch CM, Cohen SB, Jordan LC, Robertson PG, Balian G, and Diduch DR. 2002. Transforming growth factor-beta in calcium alginate beads for the treatment of articular cartilage defects in the rabbit. Arthroscopy 18:892-900. 10.1053/jars.2002.36117

Mori H, Kondo E, Kawaguchi Y, Kitamura N, Nagai N, lida H, and Yasuda K. 2013. Development of a salmon-derived crosslinked atelocollagen sponge disc containing osteogenic protein-1 for articular cartilage regeneration: In vivo evaluations with rabbits. BMC Musculoskeletal Disorders 14. 10.1186/1471-2474-14-174

Mrosek EH, Schagemann JC, Chung HW, Fitzsimmons JS, Yaszemski MJ, Mardones RM, O'Driscoll SW, and Reinholz GG. 2010. Porous tantalum and poly-epsilon-caprolactone biocomposites for osteochondral defect repair: preliminary studies in rabbits. Journal of Orthopaedic Research 28:141-148. 10.1002/jor.20983

Reinholz GG, Lu L, Saris DB, Yaszemski MJ, and O'Driscoll SW. 2004. Animal models for cartilage reconstruction. Biomaterials 25:1511-1521. 10.1016/S0142-9612(03)00498-8

Reyes R, Delgado A, Sanchez E, Fernandez A, Hernandez A, and Evora C. 2012. Repair of an osteochondral defect by sustained delivery of BMP-2 or TGFbeta1 from a bilayered alginate-PLGA scaffold. Journal of Tissue Engineering and Regenerative Medicine. 10.1002/term.1549

Reyes R, Delgado A, Solis R, Sanchez E, Hernandez A, Roman JS, and Evora C. 2014. Cartilage repair by local delivery of transforming growth factor-beta1 or bone morphogenetic protein-2 from a novel, segmented polyurethane/polylactic- co-glycolic bilayered scaffold. Journal of Biomedical Materials Research Part A 102:1110-1120. 10.1002/jbm.a.34769

Reyes R, Pec MK, Sanchez E, del Rosario C, Delgado A, and Evora C. 2013. Comparative, osteochondral defect repair: Stem cells versus chondrocytes versus bone morphogenetic protein-2, solely or in combination. European Cells and Materials 25:351-365.

Richter W. 2009. Mesenchymal stem cells and cartilage in situ regeneration. Journal of Internal Medicine 266:390-405. 10.1111/j.1365-2796.2009.02153.x

Rutgers M, van Pelt MJ, Dhert WJ, Creemers LB, and Saris DB. 2010. Evaluation of histological scoring systems for tissue-engineered, repaired and osteoarthritic cartilage. Osteoarthritis Cartilage 18:12-23. 10.1016/j.joca.2009.08.009

Seo SJ, Mahapatra C, Singh RK, Knowles JC, and Kim HW. 2014. Strategies for osteochondral repair: Focus on scaffolds. J Tissue Eng 5:2041731414541850. 10.1177/2041731414541850

Sloff M, Simaioforidis V, de Vries R, Oosterwijk E, and Feitz W. 2014. Tissue Engineering of the Bladder-Reality or Myth? A Systematic Review. The Journal of Urology 192:1035-1042. 10.1016/j.juro.2014.03.116 
841

842

843

844

845

846

847

848

849

850

851

852

853

854

855

856

857

858

859

860

861

862

863

864

865

866

867

868

869

870

871

872

873
Solchaga LA, Yoo JU, Lundberg M, Dennis JE, Huibregtse BA, Goldberg VM, and Caplan Al. 2000. Hyaluronan-based polymers in the treatment of osteochondral defects. Journal of Orthopaedic Research 18:773-780. 10.1002/jor.1100180515

Stanish WD, McCormack R, Forriol F, Mohtadi N, Pelet S, Desnoyers J, Restrepo A, and Shive MS. 2013. Novel scaffold-based BST-CarGel treatment results in superior cartilage repair compared with microfracture in a randomized controlled trial. The Journal of Bone and Joint Surgery 95:1640-1650. 10.2106/JBJS.L.01345

Sukegawa A, Iwasaki N, Kasahara Y, Onodera T, Igarashi T, and Minami A. 2012. Repair of rabbit osteochondral defects by an acellular technique with an ultrapurified alginate gel containing stromal cell-derived factor-1. Tissue Engineering Part A 18:934-945. 10.1089/ten.TEA.2011.0380

Tamai N, Myoui A, Hirao M, Kaito T, Ochi T, Tanaka J, Takaoka K, and Yoshikawa H. 2005. A new biotechnology for articular cartilage repair: Subchondral implantation of a composite of interconnected porous hydroxyapatite, synthetic polymer (PLA-PEG), and bone morphogenetic protein-2 (rhBMP-2). Osteoarthritis Cartilage 13:405-417. 10.1016/j.joca.2004.12.014

ter Riet G, Korevaar DA, Leenaars M, Sterk PJ, Van Noorden CJ, Bouter LM, Lutter R, Elferink RP, and Hooft L. 2012. Publication bias in laboratory animal research: a survey on magnitude, drivers, consequences and potential solutions. PLoS One 7:e43404. 10.1371/journal.pone.0043404

van der Linden MH, Saris D, Bulstra SK, and Buma P. 2013. Treatment of cartilaginous defects in the knee: recommendations from the Dutch Orthopaedic Association. Nederlands Tijdschrift voor Geneeskunde 157:A5719.

Wakitani S, Goto T, Pineda SJ, Young RG, Mansour JM, Caplan Al, and Goldberg VM. 1994. Mesenchymal cell-based repair of large, full-thickness defects of articular cartilage. The Journal of Bone and Joint Surgery 76:579-592.

Xie A, Nie L, Shen G, Cui Z, Xu P, Ge H, and Tan Q. 2014. The application of autologous plateletrich plasma gel in cartilage regeneration. Molecular Medicine Reports 10:16421648. 10.3892/mmr.2014.2358

Yao X, Ma X, and Zhang Z. 2000. Chondrocyte allografts for repair of full-thickness defects in the condylar articular cartilage of rabbits. The Chinese Journal of Dental Research 3:24-30.

Zhou M, and Yu D. 2014. Cartilage tissue engineering using PHBV and PHBV/Bioglass scaffolds. Molecular Medicine Reports 10:508-14 10.3892/mmr.2014.2145 